\title{
Sulfate-Based Laxative BLI850
}

National Cancer Institute

\section{Source}

National Cancer Institute. Sulfate-Based Laxative BLI850. NCI Thesaurus. Code C80080.

An oral laxative containing sodium sulfate, potassium sulfate, magnesium sulfate and sucralose. Oral sulfate-based laxative BLI850 exhibits osmotic activity, attracting water into the intestinal tract from tissues and increasing the volume and the water content of the stool; gastrointestinal motility is stimulated, resulting in defecation. Sucralose, an artificial sweetener, may contribute to the laxative effect. 